\title{
Metadata and its applications in the digital library : approaches and practices
}

\author{
Jia Liu \\ Libraries Unlimited \\ Westport (CT), USA \\ 2007 \\ $\mathrm{xx}+192 \mathrm{pp}$. \\ ISBN 978-1-159158-206-6 \\ £22.95 \\ George Macgregor \\ Information Strategy Group, Liverpool Business School \\ Liverpool John Moores University
}

Keywords metadata, digital libraries, cataloguing

Over recent years there have emerged numerous monographs on the topic of metadata (see for example: Caplan, 2003; Foulonneau and Riley, 2008; Haynes, 2004; Hillman and Westbrooks, 2004; Intner et al., 2005; Smiraglia, 2005). Each has been very much welcomed and - given that developments in metadata have accelerated with the advent of XML - every addition to the metadata canon has been necessary. This is particularly the case for the practitioner who wishes to keep pace with the rapid changes but can ill afford the time to peruse the relevant journals or websites, or participate on the multitude of email discussion lists, wikis or blogs. It is therefore extremely disappointing that Metadata and its applications fails to build upon previous contributions or deliver anything new for the reader.

Liu divides her book into two parts. The first part ('Metadata in general') encompasses an introduction to metadata, metadata types (e.g. descriptive, administrative, structural, etc.), metadata formats and encoding schemes (e.g. Dublin Core, MARC, METS, EAD, etc.), and metadata implementation matters (e.g. application profiles, metadata schema registries, harvesting, etc.). The second part ('Metadata projects and their applications in the digital library') is arguably what distinguishes her text from others; covering the work of various metadata projects and the application of metadata within digital library contexts.

Although purporting to be cutting edge, the strongest aspects of Metadata and its applications are actually to be found in the 'bread and butter' areas of metadata. Liu provides excellent introductory chapters on the functions of metadata and metadata types, including the author's own and useful metadata typology. Good worked examples of metadata schema in a variety of serialisations (e.g. XML, RDF/XML, XHTML, etc.) are sprinkled liberally throughout, as are data models and system screenshots. Unfortunately, whilst all chapters appear well referenced - at least at first glance - very few of them are published after 2004, with the URLs for some electronic references not checked since February 2003. And herein problems with Metadata and its applications become more apparent.

This book was published in late July 2007, but has the air of a book written five or six years ago. DSpace - released in 2002 and now widely implemented internationally - is described as a "newly developed digital repository" application. A detailed discussion of MARCXML is strangely shunned in favour of MARC21, a standard rarely used in digital library applications and the Metadata Object Description Schema (MODS) - a standard widely acknowledged to be the 'weapon of choice' for digital library applications (ALA, 2007) - is mentioned in a few passing sentences in relation to MARC. That Liu addresses metadata packaging is to her credit; but unfortunately only the Metadata Encoding and Transmission Standard (METS) is covered (no mention of MPEG-21 DIDL or IMS-CP is made, for example), and even here the reader is unlikely to gain any insights not already gleaned from website primers. The lack of any dedicated space to MARCXML and MODS is all the more perplexing since the latter was developed to complement the descriptive metadata section (dmdSec) of METS. MARCXML has also been embraced by high-profile digital library programmes, such as the Los Alamos National Lab Research Library (Goldsmith and Knudson, 2006). To compound bewilderment, a bizarre book 
structure means that the discussion of complex XML-based schema occurs prior to any overview of XML itself. Whilst the numbered sections make the book conducive to quick consultation thus minimising the confusion that such cart before the horse-isms might cause, it adds to the overall perception that Metadata and its applications has not been well considered.

The lack of currency also usurps the unique selling point of Liu's book: the foray into metadata reality; exploring the deployment of metadata within digital library applications, as well examining metadata research and development projects. Many of the projects covered emerged in the mid-1990s. Even where more current or ongoing projects are examined (e.g. Oxford Digital Library), the stale references prevail and little more than two pages is devoted. But perhaps most disappointing is the lack of linkage between parts one and two. Each stands in virtual isolation. Topics in part one like, say, application profiles or METS, could usefully have been extrapolated by the case studies in part two. In point of fact, Oxford Digital Library is using METS, but the reader gets little insight into the multifarious issues surrounding complex digital object management and instead is treated to a brief project overview.

In truth it is difficult to discern whether all these slips are serious or not. This is partly because there is so much dubiety as to what Metadata and its applications is supposed to be. The text is pitched as "offering a state-of-the-art analysis of metadata's major theoretical issues and most exemplary practices"; yet, no analysis actually takes place and most of the proceedings are introductory, pitched more at the novice practitioner or the aspiring student digital librarian. In this respect Metadata and its applications is essentially a basic metadata introduction, supplemented with an overview of some principal standards and a selection of prominent metadata or digital library projects. And all this is extremely depressing given Liu's credentials. A research fellow at the Alexander von Humboldt Foundation, Liu was a PhD student at Peking University before becoming a lecturer at the Department of Information Management at Peking University. She is currently a visiting scholar at the Faculty of Information Studies, University of Toronto, and has a long history at the forefront of metadata research.

Like other additions to the metadata canon, Jia Liu's book is not wholly unwelcome, carving a niche for itself by focusing on the deployment of metadata within digital library applications; however, problems with the book remit and the overall execution may mean that it is not as welcome on the bookshelf as others might be. Olympians need not apply, and the novice practitioner or student would probably be better served by alternatives available from the growing metadata canon.

\section{References}

ALA. (2007), Using metadata standards in digital libraries: implementing METS, MODS, PREMIS and MIX, LITA Standards Interest Group - American Library Association Annual Conference 2007, available: http://www.loc.gov/standards/mods/presentations/litaprogram-an2007.html (accessed 21 January 2008)

Caplan, P. (2003), Metadata fundamentals for all librarians, American Library Association, Chicago.

Foulonneau, M. and Riley, J. (2008), Metadata for digital resources: implementation, systems design and interoperability, Chandos Publishing Ltd., Oxford.

Goldsmith, B. and Knudson, F. (2006), Looking back, looking forward: a metadata standard for LANL's aDORe repository, Proceedings of the 6th ACM/IEEE-CS Joint Conference on Digital libraries, Chapel

Hill, ACM, New York, pp.272 - 273, available at:

http://portal.acm.org/ft gateway.cfm?id=1141814\&type=pdf\&coll=portal\&dl=ACM\&CFID=51016048 $\underline{\text { \&CFTOKEN }=75960609}$ (accessed 21 January 2008)

Haynes, D. (2004), Metadata for information management and retrieval, Facet Publishing, London.

Hillman, D.I. and Westbrooks, E.L. (2004), Metadata in practice, American Library Association, Chicago. 
Intner, S.S., Lazinger, S.S. and Weihs, J. (2005), Metadata and its impact on libraries, Libraries Unlimited, Westport (USA).

Smiraglia, R.P. (Ed.) (2005), Metadata : a cataloger's primer, Haworth Information Press, Binghamton (NY). 